\title{
Characterization of stability of ceramic suspension for slurry introduction in inductively coupled plasma optical emission spectrometry and application to aluminium nitride analysis
}

\author{
Zheng Wang, ${ }^{a b}$ Zheming Ni, ${ }^{c}$ Deren Qiu, ${ }^{b}$ Guangyi Tao ${ }^{a}$ and Pengyuan Yang*b \\ ${ }^{a}$ Shanghai Institute of Ceramics, Graduate School of Chinese Academy of Sciences, \\ Shanghai 200050, China. E-mail: wangzheng@mail.sic.ac.cn \\ ${ }^{\boldsymbol{b}}$ Department of Chemistry, Fudan University, Shanghai 200433, China. \\ E-mail:pyyang@fudan.edu.cn \\ ${ }^{c}$ Research Center for Eco-Environmental Sciences, Academia Sinica, P.O. Box 2871, \\ Beijing 100085, China
}

Received 11th October 2004, Accepted 20th January 2005

First published as an Advance Article on the web 15th February 2005

Characterization of the stability of ceramic suspension for slurry introduction in inductively coupled plasma optical emission spectrometry (ICP-OES) and its application to the analysis of aluminium nitride (AlN) was reported in this paper. Stability and homogeneity suspensions of $\mu \mathrm{m}$ sized AlN and nm sized AlN, as the representatives selected, were characterized by sedimentation test, scanning electron microscope observation and zeta potential measurement. The zeta potential of the interface between the particle and the medium can be controlled by $\mathrm{pH}$ adjustment and adding $0.8 \mathrm{wt} \%$ polyethylene imine (PEI) for $\mu \mathrm{m}$ size $\mathrm{AlN}$ or $2.0 \mathrm{wt} \%$ PEI for nm size AlN, respectively. The analytical results obtained via slurry introduction were compared with those via nebulization of aqueous solutions prepared by fusion digestion. The nm size AlN can be directly analyzed by using slurry nebulization ICP-OES with aqueous standards calibration, and the impurities $\mathrm{Cu}, \mathrm{Fe}, \mathrm{Mg}, \mathrm{Mn}$, etc. in $\mu \mathrm{m}$ size $\mathrm{AlN}$ can also be determined by slurry method, but the results of some elements with high boiling points, such as $\mathrm{Si}$, Y, etc., show deviation from the reference values.

\section{Introduction}

The development and production of new ceramic materials requires sensitive analytical methods for the determination of impurities at trace levels. Inductively coupled plasma optical emission spectrometry (ICP-OES) is nowadays the main method for the trace analysis of the inorganic elements. In routine work, the sample should be prepared in the form of a solution and a nebulization technique for sample introduction is used. However, ceramics such as $\mathrm{Al}_{2} \mathrm{O}_{3}, \mathrm{TiO}_{2}, \mathrm{SiC}, \mathrm{Si}_{3} \mathrm{~N}_{4}$ and $\mathrm{AlN}$ are difficult to digest into solution even using microwaveassisted digestion. Fusion dissolution causes a large factor of dilution and severe contamination from the fusion reagents and vessels. The critical requirements of high purity of the fusion reagents and time consumption limit it as a routine procedure for the analysis of these materials. An alternative approach is to prepare the sample as a slurry, which has been applied in GFAAS for a long time. Several papers of ceramics analysis by ICP-OES using slurry sample introduction have been reported. ${ }^{1-6}$ For the control of the purity of products for ceramics, determination of impurities is necessary. Technically, these materials must be of small grain size, even nanometer size. Accordingly, the slurry nebulization technique can be directly analyzed without the need for grinding, and therefore avoids time consumption and contamination.

A highly stable and homogeneous slurry is required in order to provide precise and accurate analytical results. In an excellent book, Conley ${ }^{7}$ described various aspects of dispersion of suspensions. Farinas et $a l^{8}$ mentioned that the two main factors determining the capability of the slurry technique are the particle size of the starting powders and the stability and homogeneity of the suspension. They investigated the effect of the stability and homogeneity of the suspension on line intensity and measurement precision in ICP-OES. Varga et al. ${ }^{9}$ investigated the effect of $\mathrm{pH}$ of aqueous ceramic suspension on colloidal stability and precision of analytical measurements using slurry nebulization ICP-OES. Ebdon et al. ${ }^{10}$ reviewed slurry nebulization for plasmas and reported that preparation of a stable and homogeneous suspension was an indispensable step prior to slurry nebulization in plasma: they pointed out that there are only a few in-depth studies in the references regarding the stabilizing effect of dispersants.

It is also noticeable that although analytical atomic spectrometry has been used for the analysis of many advanced ceramic materials, details of sample preparation are very scarcely reported. ${ }^{11}$ On the other hand, one should be more cautious when the slurry nebulization ICP-OES technique was used to analyze ceramic powders because the transport and nebulization efficiency are strongly influenced by the stability and homogeneity of the aqueous slurries., ${ }^{9,12}$

Recently, Wang et al. ${ }^{13}$ reported the determination of metal impurities in titanium dioxide using slurry sample introduction with axial viewing ICP-OES and showed that preparation of a stable and homogeneous aqueous slurry is vital for the determination. $\mathrm{TiO}_{2}$ aqueous suspension was stabilized by addition of polyacrylate amine $\left(\mathrm{NH}_{4} \mathrm{PAA}\right)$ dispersant.

$\mathrm{AlN}$ is one of the typical ceramics having special properties such as high thermal conductivity, low dielectric constant, high electrical resistance, low density, and a thermal expansion coefficient that matches well with that of silicon. ${ }^{14,15}$ However, its properties are significantly affected by some impurities. ${ }^{14,15}$, Using slurry nebulization for ICP-OES analysis of AlN is a reasonable selection. The preparation of a stable and homogeneous slurry is thus considered as being the first important step.

It is well known that the stability of a slurry depends substantially on the interactions between the suspended solid particles. A rapid phase separation (flocculation) occurs if the 
Van der Waals attraction overcomes the electrostatic repulsion. The $\mu \mathrm{m}$ or $\mathrm{nm}$ AlN powder suspension is unstable in simple aqueous solution. Luo et al. ${ }^{16}$ used $\mathrm{NH}_{4} \mathrm{PAA}$ as a dispersant to stabilize treated sub- $\mu \mathrm{m}$ AlN powder in aqueous solution. Castor oil has also been used as the dispersant of AIN slurry by Huang. ${ }^{17}$ Polyethylene imine (PEI), used as a universal cation dispersant, has been reported to disperse $\mathrm{SiC}^{18}$ and $\mathrm{ZrO}_{2},{ }^{19}$ but it has not been yet used to disperse AlN powder.

In this work, representative $\mu \mathrm{m}$ and nm particle sizes of AlN powder and $\mathrm{NH}_{4} \mathrm{PAA}$ and PEI dispersant were selected. Characterization of slurry stability and homogeneity was carried out. Influential factors on the stability were described. Slurry nebulization was applied to the analysis of $\mu \mathrm{m}$ size AlN and $\mathrm{nm}$ size $\mathrm{AlN}$, and analytical results were presented and discussed.

\section{Experimental}

\section{Instrumentation}

All measurements were performed on a VISTA AX ICP-OES spectrometer with axial view configuration (Varian, Australia). The sample introduction system consists of a $\mathrm{V}$-groove nebulizer and a reduced-volume Sturman-Masters type spray chamber made of poly(tetrafluorethylene) (PTFE). The operating parameters and selected analytical lines are listed in Table 1 and Table 2, respectively.

\section{Material selected and slurry preparation}

Two different sizes of AlN particle were selected for testing. The $\mu \mathrm{m}$ size AlN powder was synthesized by a self-propagation high temperature synthesis method and anti-hydrolysis treated simultaneously by using phosphoric acid (Shanghai Institute of Ceramics, CAS, China). The other one was nm size AIN (Hefei Kiln Nanometer Technology Development Co. Ltd, China).

Slurry was prepared by weighing AlN sample and then transferring it into a $100 \mathrm{ml}$ volumetric flask containing dispersant $\mathrm{NH}_{4} \mathrm{PAA}$ (Aldrich Chemical Co., USA) or PEI (DH Laboratory, UK). The $\mathrm{pH}$ was adjusted to the desired value with $\mathrm{HCl}$ or aqueous $\mathrm{NH}_{3}$. Just before nebulization, the slurry was agitated in an ultrasonic bath for 15 min to ensure a stable and good dispersion.

\section{Characterization and dispersion of slurries}

Zeta potential measurement. The zeta potential of the suspensions was measured by Zeta-Plus Analyser (Brookhaven Instruments, USA). Suspensions were prepared by adding different amounts of dispersant to AlN powders (0.01 wt.\%). The suspensions were mixed ultrasonically just before zeta potential measurement.

Table 1 Instrumentation and operating conditions

\begin{tabular}{ll}
\hline Spectral range & $167-785 \mathrm{~nm}$ \\
Viewing & Axial \\
Rf generator & $40 \mathrm{MHz}$ \\
Nebulizer & V-groove \\
Torch & All-quartz \\
Injector tube diameter & $2.3 \mathrm{~mm}$ \\
Power & $1.25 \mathrm{~kW}$ \\
Plasma flow & $151 \mathrm{~min}^{-1}$ \\
Auxiliary flow & $1.51 \mathrm{~min}^{-1}$ \\
Nebulizer flow & $0.801 \mathrm{~min}^{-1}$ \\
Replicate read time & $3 \mathrm{~s}$ \\
Replicates & 3 \\
Instr. stabilization delay & $20 \mathrm{~s}$ \\
Sample uptake delay & $15 \mathrm{~s}$ \\
Sample uptake rate & $0.8 \mathrm{ml} \mathrm{min}$ \\
\hline
\end{tabular}

Table 2 Selected analytical lines

\begin{tabular}{ll}
\hline Element & Spectral lines/nm \\
\hline $\mathrm{Cr}$ & 205.560 \\
$\mathrm{Cu}$ & 324.754 \\
$\mathrm{Fe}$ & 238.204 \\
$\mathrm{Mg}$ & 279.553 \\
$\mathrm{Mn}$ & 257.610 \\
$\mathrm{Ni}$ & 231.604 \\
$\mathrm{P}$ & 213.618 \\
$\mathrm{Si}$ & 288.158 \\
$\mathrm{Ti}$ & 334.188 \\
$\mathrm{Y}$ & 360.074 \\
$\mathrm{Zr}$ & 339.198 \\
\hline
\end{tabular}

Sedimentation measurement. Settling experiments were conducted using a $2 \mathrm{wt} \%$ AlN suspension with various amounts of $\mathrm{NH}_{4} \mathrm{PAA}$ or PEI as the dispersant. The suspensions were ultrasonically treated for $30 \mathrm{~min}$, and then settled in $10 \mathrm{ml}$ graded tubes. The readings of sedimentation volume were recorded after two weeks.

\section{SEM observation and particle size determination}

The suspensions were settled on a copper slice under gravity until a thin sediment film was formed. The sediment was then dried in the air and observed with a scanning electron microscope (SEM) (JSM-6700F, Japan). To obtain high-quality micrographs, the sediments were coated with a thin gold layer prior to measurement. The particle size of $\mu \mathrm{m}$ AlN was determined by SICAS-4800 Photo-Sedimentometry (Shanghai Institute of Ceramics, CAS). The particle size of nm AlN is gained from the SEM image. The distribution of particles is illustrated in Figs. 1 and 2, respectively.

\section{Reagents and samples}

All reagents were of guarantee grade. The solutions were prepared with Milli-Q water $(18 \mathrm{M} \Omega \mathrm{cm})$. Calibration curves were established by use of aqueous standards. The multielement standards were prepared from $1000 \mathrm{mg} \mathrm{l}^{-1}$ aqueous standards (Shanghai Institute of Measurement and Testing Technology, Shanghai, China). The blank and standards were prepared containing same amount of dispersant.

The reference data were taken from a fusion prepared clear solution of the same sample. Sample $(0.1 \mathrm{~g})$ was mixed with an alkaline flux ( $2 \mathrm{~g}, \mathrm{Na}_{2} \mathrm{CO}_{3}+$ borax $)$ and fused in a Pt crucible for $30 \mathrm{~min}$. After cooling to room temperature, water was

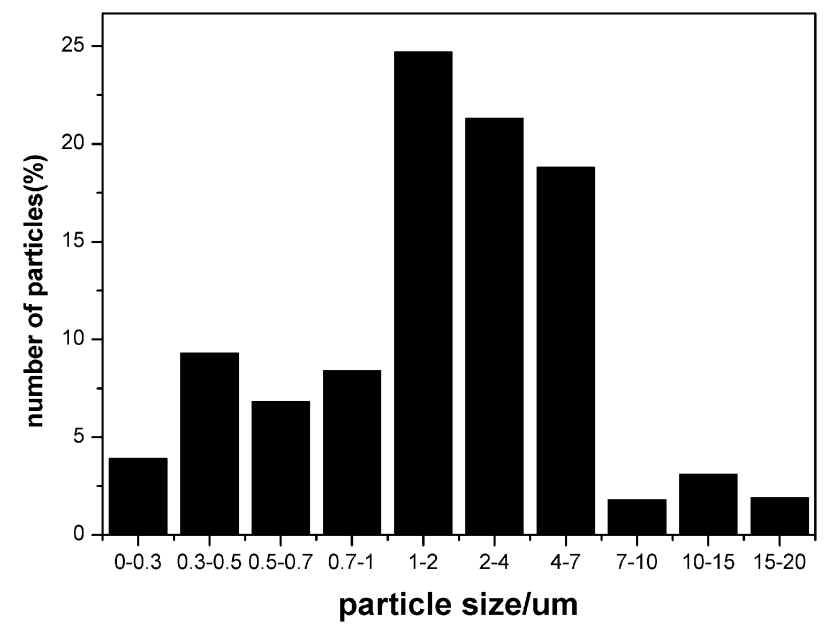

Fig. 1 Aluminium nitride distribution diagram for $\mu \mathrm{m}$ particle size of slurry (determined by photosedimentometry). 

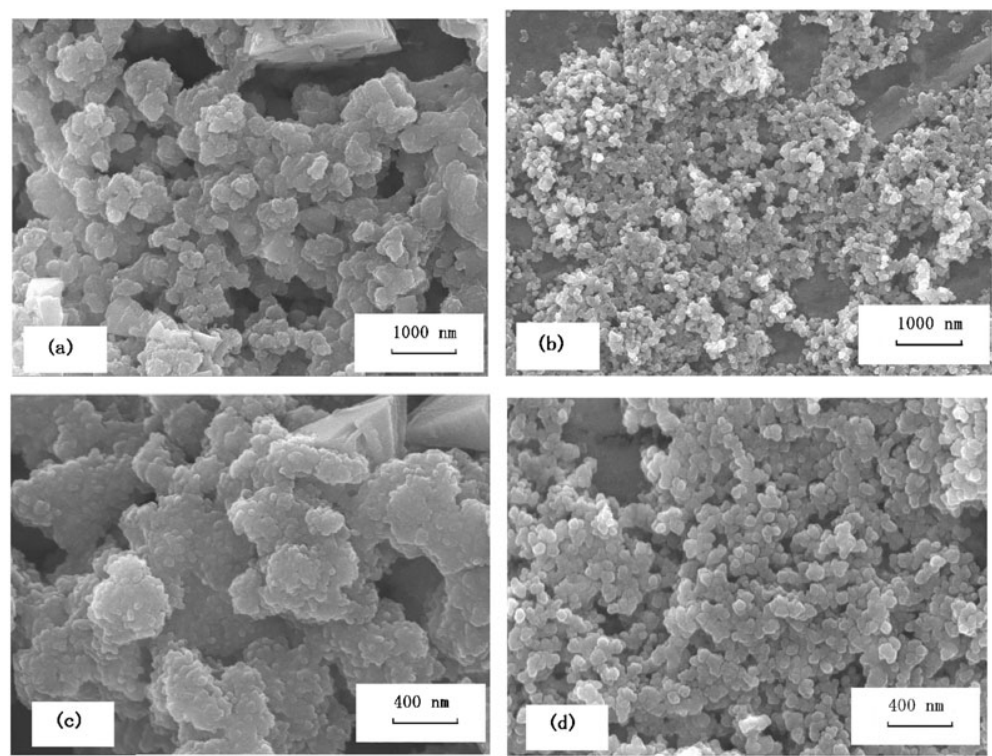

Fig. 2 SEM micrographs of sediments resulting from 2 wt. \% nm AlN suspension at $\mathrm{pH} 6.0$ : (a) without dispersants $\times 20000$; (b) with dispersants $\times 20000$; (c) without dispersants $\times 50000$; (d) with dispersants $\times 50000$.

added to cover the fusion cake and the sample was left overnight. The dissolved sample solution was transferred into a polyethylene volume flask and made up to a volume of $100 \mathrm{ml}$ with water.

\section{Results and discussion}

\section{Characterization and optimisation of suspension}

The aim of the characterization of the slurry is to establish the optimised conditions for preparing a stable and homogeneous suspension. As a representative, AlN slurry was characterized with particle size distribution, sedimentation test and zeta potential measurement. Dispersant and its appropriate amount were taken on the basis of the characterized results. The stability of the suspension under optimised conditions was finally experimentally verified by the normalized spectroscopic signals versus time of slurry introduction.

The distribution data were determined by the photo-sedimentation experiments. Fig. 1 shows that most partitions of the $\mu \mathrm{m}$-AlN powder are smaller than $7 \mu \mathrm{m}$. The mean diameter is $1.9 \mu \mathrm{m}$. Fig. 2 shows the morphology of the nm AlN particles during SEM observation. It shows that nm particle size of AlN is far smaller than $1 \mu \mathrm{m}$. Fig. 3 shows the relationship of the sediment volume of $\mu \mathrm{m}$-AlN suspensions versus the concentration of $\mathrm{NH}_{4} \mathrm{PAA}$ or PEI after resting for two weeks at $\mathrm{pH} 6.0$.

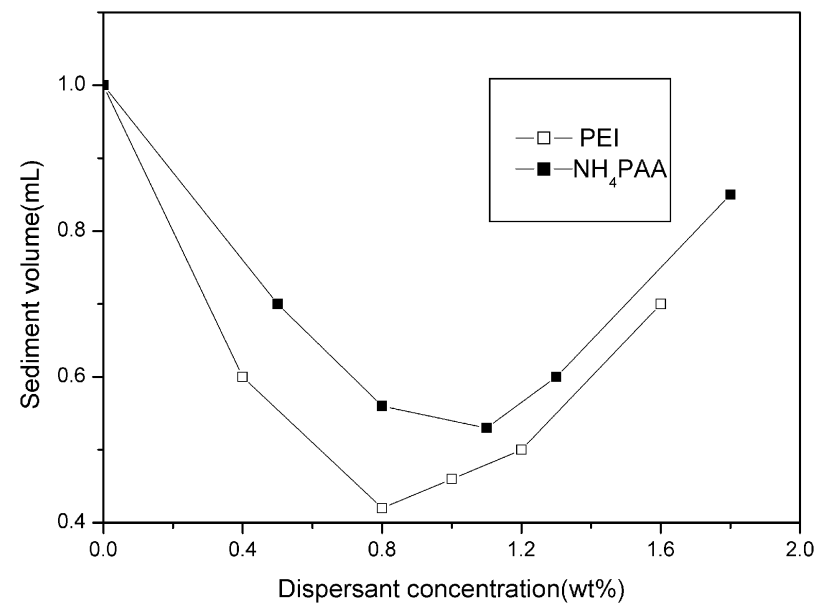

Fig. 3 Sediment volume of $2 \mathrm{wt} \% \mu \mathrm{m}$ aluminium nitride suspension as a function of dispersant concentration after two weeks.
As is shown in Fig. 3, the sediment volume reached a minimum at $0.8 \%$ for PEI and $1.0 \%$ for $\mathrm{NH}_{4} \mathrm{PAA}$, respectively. The starting point, namely a dispersant concentration of zero, was the simple aqueous slurry prepared without addition of PEI or $\mathrm{NH}_{4} \mathrm{PAA}$. It indicated that the aqueous $\mu \mathrm{m}$ size AIN suspensions with $0.8 \% \mathrm{PEI}$ is in a well-stable state. It also shows that the stability effect of PEI is better than that of $\mathrm{NH}_{4} \mathrm{PAA}$. Therefore, PEI was selected as the dispersant that has been investigated in detail.

Farinas et al. $^{7}$ have discussed the relationship between stability and the zeta potential. In this paper, we characterized the stability via measuring the zeta potential of the system to optimise the conditions for a stable suspension. Fig. 4 presents the dependence of $\mathrm{pH}$ on the zeta potential of $\mu \mathrm{m}$ particle size AlN slurry at different PEI concentrations. The zeta potential of the slurry has an important effect on the dispersion state of particles in suspensions, which represents the electrostatic interaction between particles in the dispersion. The presence of too much dispersant also leads to instability, since the electrostatic double layer is compressed by the large amount of dispersant. Therefore, it is vital to determine the isoelectric point (IEP) for the preparation of stable slurry. In the case where the IEP is determined, the appropriate $\mathrm{pH}$ region for slurry stability is experimentally determined in which suffi-

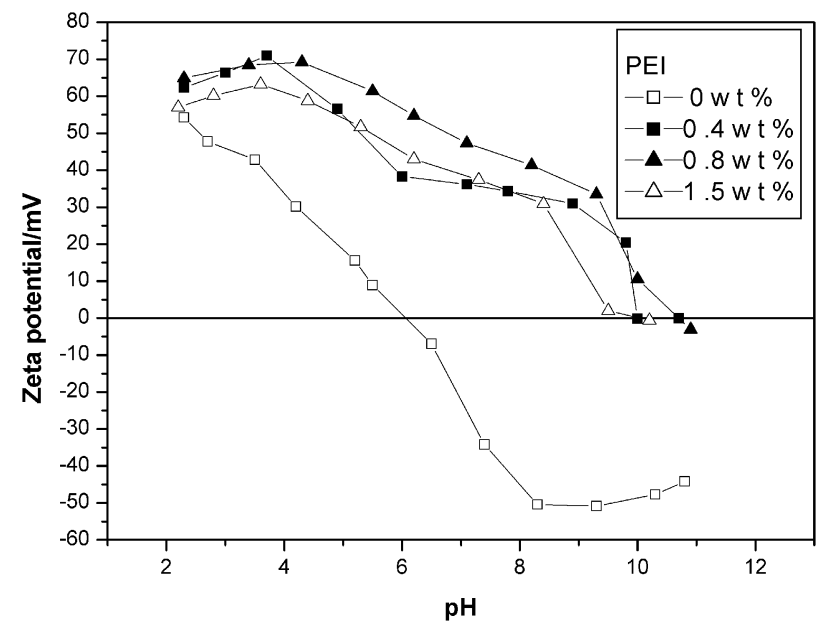

Fig. 4 Profiles of zeta potential versus $\mathrm{pH}$ for $\mu \mathrm{m}$ particle size AlN slurry at different PEI concentrations. $(\square, 0 \mathrm{wt} \% ; \boldsymbol{\square}, 0.4 \mathrm{wt} \% ; \boldsymbol{\Delta}, 0.8$ $\mathrm{wt} \% ; \triangle, 1.5 \mathrm{wt} \%$ ). 
ciently high charged surface generates a strongly repulsive, so that the slurry is stable. The isoelectric points are found near $\mathrm{pH}$ 6.0 for no dispersant. Adding PEI moved the IEP towards the alkaline region. The experiment shows that the zeta potential at $\mathrm{pH} 5$ ascends from about $20 \mathrm{mV}$ for simple aqueous slurry to about $60 \mathrm{mV}$ for a spiked slurry. From Fig. 4 it can be found that $0.8 \mathrm{wt} \% \mathrm{PEI}$ is the appropriate amount for dispersing the $\mu \mathrm{m}$ size AlN powder and the zeta potential was essentially much

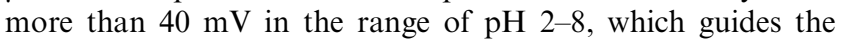
appropriate $\mathrm{pH}$ range conditions for slurry preparation. The result agreed well with that of sedimentation test.

The relationship of the zeta potential of a $\mathrm{nm}$ particle size AlN slurry versus $\mathrm{pH}$ at different PEI concentrations is plotted in Fig. 5 The IEP of nm particle AlN is $\mathrm{pH} 8.9$, different from the above mentioned IEP of $\mu \mathrm{m}$ size AlN. The $\mu \mathrm{m}$ size AlN powder has been treated with phosphoric acid in order to limit AlN hydrolysis, whereas nm particle AlN has not been treated by acid. Similarly to the $\mu \mathrm{m}$ size AIN, the IEP of nm particle AlN also moved towards a basic region (about $\mathrm{pH} 10.2$ ). The zeta potential was increased initially with the increase of dispersant concentration, then a plateau value was achieved. The zeta potential at $\mathrm{pH} 6$ changes from $30 \mathrm{mV}$ for without any dispersant to $40 \mathrm{mV}$ for $2 \mathrm{wt} \%$ PEI. The experiments show that $2 \mathrm{wt} \%$ PEI is the appropriate amount for dispersing the $\mathrm{nm}$

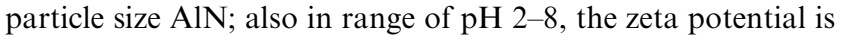
almost kept constant. The $\mathrm{nm}$ particle size AIN has a large specific surface area and requires more PEI to disperse the particles, compared with $\mu \mathrm{m}$ particle size of AlN.

The dispersing effect of the PEI is also verified by comparing the SEM micrographs of sediments resulting from $2 \mathrm{wt} \% \mathrm{~nm}$ size AlN suspension without dispersant at pH 6 in Fig. 2 (Fig. 2(a), (c), magnification of $\times 20000$ and $\times 50000$, respectively) and with $2 \mathrm{wt} \%$ dispersant PEI at pH 6 (Fig. 2(b), (d), magnification of $\times 20000$ and $\times 50000$, respectively). Obvious differences can be observed. Large flocs are observed in the sediments of suspensions without PEI, while, particles of the sediments with $2 \mathrm{wt} \%$ PEI are not associated with each another, confirming that particles are well dispersed in the suspension.

\section{Effect of slurry stability on the line intensity}

Preparing the AIN slurries in aqueous solution without dispersant was unsuitable owing to the agglomeration or flocculation effect. When the AIN powder was simply dispersed into the water, flocculation of the powder happened quickly. Slurry stability was experimentally tested as shown in Figs. 6 and 7. The two particle sizes of AlN slurry with no dispersant at IEP

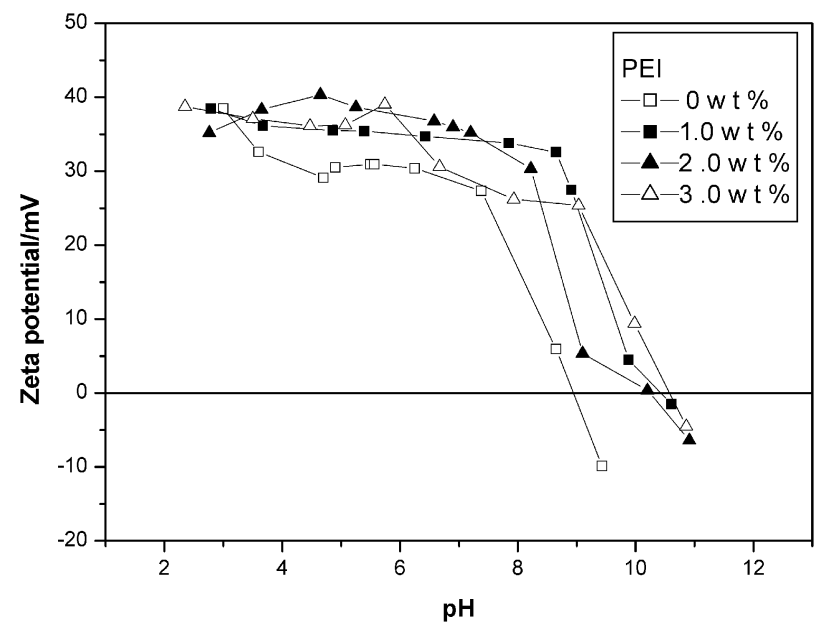

Fig. 5 Profiles of zeta potential versus $\mathrm{pH}$ for $\mathrm{nm}$ particle size AlN slurry at different PEI concentrations. $(\square, 0 \mathrm{wt} \% ; \mathbf{\square}, 1.0 \mathrm{wt} \% ; \boldsymbol{\Delta}, 2.0$ $\mathrm{wt} \% ; \triangle, 3.0 \mathrm{wt}^{0} \%$ ).

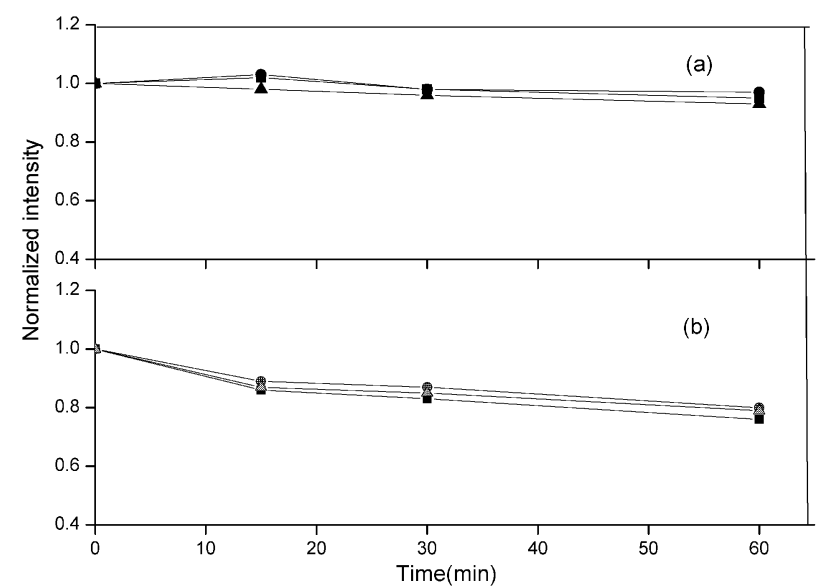

Fig. 6 Profiles of normalized intensity versus time for nm particle size AlN slurry at pH 8.9 (a) with $2.0 \mathrm{wt} \%$ PEI dispersant and (b) without dispersant $(\boldsymbol{A l}$; $\mathrm{Fe} ; \boldsymbol{\Delta} \mathrm{Si})$.

(zeta potential is zero) display unstable character. The normalized intensities of matrix $\mathrm{Al}$ and impurities $\mathrm{Fe}$ and Si obviously decreased with time, as is shown in Figs. 6(b) and 7(b). Comparatively, the nm AIN slurry with dispersant PEI (2.0 $\mathrm{wt} \%$ ) (Fig. 6(a)) displays stable line intensities in a period of $60 \mathrm{~min}$. The $\mu \mathrm{m}$ size of AlN also displays stable line intensity. However, the intensity of matrix $\mathrm{Al}$ and impurities $\mathrm{Fe}, \mathrm{Si}$ decreased with the time after 15 min (Fig. 7(a)). Normally, the analysis was soon followed by the preparation of the slurry; however, it is customary to subject the slurry to an ultrasonic bath for $15 \mathrm{~min}$ just before nebulization to ensure that is well dispersed and stable.

\section{Atomization difference between $\mathrm{nm}$ and $\mu \mathrm{m}$ size AIN}

The rigorous studies reported that slurry particles larger than 5 $\mu \mathrm{m}$ (in some reports $2 \mu \mathrm{m}$ ) do not reach the plasma and result in a loss of signal. ${ }^{10}$ In the case of the routine procedure with nebulization of a clear aqueous solution, aerosol behaviour of transportation and atomization in the central channel of the ICP torch has been thoroughly studied. However, slurry particles have a quite different behaviour from aerosol: evaporation of the particles may be the bottleneck. Raeymaekers et al. ${ }^{1}$ and Van Borm et al. ${ }^{20}$ studied the influence of particle size of $\mathrm{Al}_{2} \mathrm{O}_{3}$ and $\mathrm{SiO}_{2}$ on transportation and atomization efficiencies. The analytical results with slurry nebulization for nm size AlN (from Hefei Kiln Nanometer Technology Development Co. Ltd) were listed in Table 3, together with the

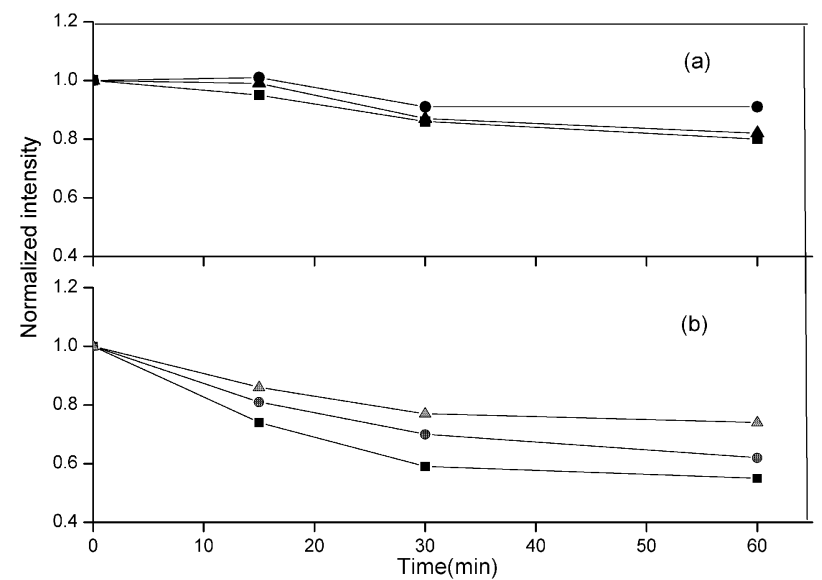

Fig. 7 Profiles of normalized intensity versus time for $\mu \mathrm{m}$ particle size AIN slurry at pH 6.0 (a) with $0.8 \mathrm{wt} \%$ PEI dispersant and (b) without dispersant ( $\mathbf{A l}$; $\mathrm{Fe} ; \boldsymbol{\Delta} \mathrm{Si})$. 
Table 3 Mean value and precision for determination of micro and trace elements in $\mathrm{nm}$ particle size $\mathrm{A}_{1} \mathrm{~N}^{a}$

\begin{tabular}{lcc}
\hline Element & Alkaline fusion $/ \mu \mathrm{g} \mathrm{g}^{-1}$ & Slurry method $/ \mu \mathrm{g} \mathrm{g}^{-1}$ \\
\hline $\mathrm{Cr}$ & $295 \pm 5$ & $288 \pm 10$ \\
$\mathrm{Fe}$ & $425 \pm 6$ & $429 \pm 4$ \\
$\mathrm{Mg}$ & $6 \pm 3$ & $3 \pm 2$ \\
$\mathrm{Mn}$ & $14 \pm 2$ & $13 \pm 2$ \\
$\mathrm{Ni}$ & $144 \pm 12$ & $150 \pm 8$ \\
$\mathrm{Si}$ & $610 \pm 16$ & $619 \pm 15$ \\
$\mathrm{Ti}$ & $37 \pm 3$ & $40 \pm 4$ \\
${ }^{a}$ Mean value $\pm \mathrm{SD}(n=5)$. & \\
\hline
\end{tabular}

Table 4 Mean value and precision for determination of micro and trace elements in $\mu \mathrm{m}$ particle size $\mathrm{A}_{1} \mathrm{~N}^{a}$

\begin{tabular}{lcc}
\hline Element & Alkaline fusion $/ \mu \mathrm{g} \mathrm{g}^{-1}$ & Slurry method $/ \mu \mathrm{g} \mathrm{g}^{-1}$ \\
\hline $\mathrm{Cu}$ & $28 \pm 2$ & $29 \pm 2$ \\
$\mathrm{Fe}$ & $808 \pm 16$ & $778 \pm 20$ \\
$\mathrm{Mg}$ & $16 \pm 4$ & $19 \pm 3$ \\
$\mathrm{Mn}$ & $10 \pm 1$ & $6 \pm 3$ \\
$\mathrm{Ni}$ & $12 \pm 2$ & $12 \pm 2$ \\
$\mathrm{P}$ & $250 \pm 8$ & $249 \pm 7$ \\
$\mathrm{Si}$ & $1079 \pm 41$ & $380 \pm 21$ \\
$\mathrm{Ti}$ & $27 \pm 1$ & $15 \pm 2$ \\
$\mathrm{Y}$ & $1890 \pm 25$ & $1145 \pm 60$ \\
$\mathrm{Zr}$ & $1200 \pm 31$ & $1045 \pm 60$ \\
${ }^{a}$ Mean value & $\pm \mathrm{SD}(n=5)$. & \\
\hline
\end{tabular}

reference values expressed in terms of "alkaline fusion". The reference values were determined from the same sample prepared via fusion with pure $\mathrm{Na}_{2} \mathrm{CO}_{3}+$ borax, and the blanks have been deducted. Table 3 shows that the impurities in $\mathrm{nm}$ size AlN slurry could be directly determined with calibration of aqueous standards.

The analytical results for another sample, $\mu \mathrm{m}$ size AlN (from Shanghai Institute of Ceramics), are listed in Table 4 together with the reference values also taken from the alkaline fusion procedure described above. For $\mu \mathrm{m}$ size AlN slurry, as shown in Table 4, the results for elements $\mathrm{Cu}, \mathrm{Fe}, \mathrm{Mg}, \mathrm{Mn}, \mathrm{Ni}$ and $\mathrm{P}$ were in good accordance with the fusion method. However, the results for $\mathrm{Y}, \mathrm{Si}$ and others deviated from the reference values. In our opinion, result deviation might be chiefly attributed to incomplete evaporation of these elements with high boiling point in the $\mu \mathrm{m}$ size $\mathrm{AlN}$.

\section{Calibration techniques}

As can be seen in Table 3, the prepared slurry of nm size AlN can be directly analyzed using slurry nebulization with aqueous standards calibration. All the results were in good accordance with the reference values.

As is shown in Table 4, for $\mu \mathrm{m}$ size AlN, the analytical results of impurities $\mathrm{Cu}, \mathrm{Fe}, \mathrm{Mg}, \mathrm{Mn}$, etc., were in agreement with the reference values and can thus be accurately determined by the slurry method with aqueous standards calibration. However, the results of $\mathrm{Si}, \mathrm{Y}$, etc. obviously deviated. The method of standard additions for calibration was tested with only limited effect and no improvement occurred. The analytes added into the slurry exist in the form of ions: however, the analytes in solid particles exist in a quite different chemical form. Therefore, the evaporation and atomization behaviour of the analytes in the aqueous phase is somewhat different from that in solid particles. Moreover, the larger size of the particle, the greater the difference in behaviour. For nm size AlN, the difference is so small that it could be negligible. For the $\mu \mathrm{m}$ size particles result deviation might be mainly attributed to their high boiling point and incomplete evaporation from the larger size AIN in the plasma channel. It is not too much to say that the slurry procedure with aqueous calibration for $\mu \mathrm{m}$ particle size AlN could be acceptable as a semi-quantitative method for screening the raw material in production control.

\section{Conclusions}

1. In order to prepare a stable and homogeneous suspension for slurry nebulization of ceramic samples in ICP-OES, characterization for stability of suspension is necessary. Different sizes of the material need the addition of different optimized amounts of the dispersant and adjustment of different $\mathrm{pH}$ to achieve an appropriate zeta potential for stabilization.

2. The nm size AIN can be directly analyzed using slurry introduction ICP-OES with aqueous standards calibration.

3. Some impurities, such as $\mathrm{Cu}, \mathrm{Fe}, \mathrm{Mg}, \mathrm{Mn}$, etc., in $\mu \mathrm{m}$ size AlN can also be determined by the slurry method, but some elements with high boiling point, such as $\mathrm{Si}, \mathrm{Y}$, etc., cannot be accurately determined. The method of standard additions is not available to the slurry nebulization ICP-OES for calibration for the analysis of the $\mu \mathrm{m}$ size AlN slurry.

\section{Acknowledgements}

The authors are grateful to Shanghai Committee of Science and Technology $(0159 \mathrm{~nm} 075)$ and Shanghai Institute of Ceramics, CAS (ZRJJ 200401), for financial support.

\section{References}

1 B. Raeymaekers, T. Graule, J. A. C. Broekaert, F. Adams and P. Tschopel, Spectrochim. Acta, Part B, 1988, 43, 923.

2 T. Graule, A. V. Bohlen, J. A. C. Broekaert, E. Grallath, R. Kloekenkamper, P. Tschopel and G. Tolg, Fresenius' J. Anal. Chem., 1989, 335, 637.

3 M. Huang and X. Shen, Spectrochim. Acta, Part B, 1989, 44, 957.

4 B. Docekal, J. A. C. Broekaert, T. Graule, P. Tschopel and G. Tolg, Fresenius' J. Anal. Chem., 1992, 342, 113.

5 J. A. C. Broekaert, C. Lathen, R. Brandt, C. Pilger, D. Pollmann, P. Tschopel and G. Tolg, Fresenius' J. Anal. Chem., 1994, 349, 20.

6 G. Zaray, I. Varga and T. Kantor, J. Anal. At. Spectrom., 1994, 9, 707.

7 R. F. Conley, Practical Dispersion - A guide to understanding and formulating slurries, VCH, 1996.

8 J. C. Farinas, R. Moreo and J. M. Mermet, J. Anal. At. Spectrom., 1994, 9, 841 .

9 I. Varga, F. Csempesz and G. Zaray, Spectrochim. Acta, Part B, 1996, 51, 253.

10 L. Ebdon, M. Foulkes and K. Sutton, J. Anal. At. Spectrom., 1997, 12, 213.

11 A. Fisher, M. W. Hinds, S. N. Nelms, D. M. Penny and P. Goodall, J. Anal. At. Spectrom., 2002, 17, 1624.

12 Z. Wang, Z. Ni, D. Qiu, T. Chen, G. Tao and P. Yang, 2004 Winter Conference on Plasma Spectrochemistry, Fort Lauderdale, Florida, TP13.

13 Z. Wang, Z. Ni, D. Qiu, T. Chen, G. Tao and P. Yang, J. Anal. At. Spectrom., 2004, 19, 273.

14 A. V. Virkar, T. B. Jackson and R. A. Cutler, J. Am. Ceram. Soc., 1989, 72(11), 2031.

15 Y. Zhou, D. Wang, Y. Xi, H. Zhuang, S. Wen, J. Guo and D. Yan, J. Inorg. Mater., 1999, 14(4), 674 (in Chinese).

16 X. Luo, X. Xu, B. Zhang, W. Li and H. Zhuang, Mater. Sci. Eng. A, 2004, 368, 126.

17 H. Huang, H. Zhou and Y. Wang, J. Inorg. Mater., 2002, 17(2), 380 (in Chinese).

18 J. Sun and L. Gao, J. Eur. Ceram. Soc., 2001, 21, 2447.

19 J. Wang and L. Gao, J. Colloid Interface Sci., 1999, 216, 436.

20 W. A. H. Van Borm, J. A. C. Broekaert, R. Klockenkamper and P. Tschopel, Spectrochim. Acta, Part B, 1991, 46, 1033. 